\title{
Congestion Control in Wireless Network using TCP Friendly Rate Control (TFRC)
}

\author{
K. Rangaswamy, C. Rajabhushanam
}

\begin{abstract}
Throughout the years in wireless networks, congestion control has been widely explored. With the point of improving execution and performance in wireless network many techniques and methods have been built up over the years. It is very necessary to solve the issue of congestion control for the quick extension and execution of wireless technology. When a network link or node carries more data than its capacity then network congestion will occurs. In queueing theory and network congestion it reduces the quality of service. Normal impacts of congestion control include packet loss, blocking of new connections and queueing delay. As a result of congestion is to increment in offered load either just to a little increment or even a reduction in system throughput [1].There are many ways to control the congestion in wireless networks. We studied different techniques in this paper and also work through applying TCP friendly rate control.
\end{abstract}

Index Terms: congestion control, network, wireless..

\section{INTRODUCTION}

In wireless networks congestion control is an open and significant issue. In many past demonstration it has been shown that Transport Control Protocol (TCP) give the poor performance. The main reason behind poor performance of the TCP is that it cannot identify or detect and unable to respond appropriately at particular condition. TCP uses TCP window based ow control algorithm and that isn't reasonable for the remote channel, and because of mobility congestion collapse. In wired and wireless networks new rate based techniques have been introduced. By the absence of appropriate bandwidth estimation methods for multi-hop wireless networks, these techniques also show the poor work. It is in this way essential to enhance the capacity of the congestion control in wireless networking.

To avoid the collapse network utilize the congestion avoidance and congestion control methods. In these methods include window reduction in TCP, exponential back off in protocols such as CSMA/CA in 802.11 and the similar CSMA/CD in the original Ethernet, and reasonable queueing in gadgets, for example, switches and system switches. In other approaches some congestion control have priority methods in which the packet which have higher priority take place in front of others and the unequivocal distribution of

Revised Manuscript Received on July 22, 2019

K. Rangaswamy, Research Scholar, Dept. of C.S.E, Bharath Institute of Higher Education and Research, Chennai, Tamilnadu, India. Email: rangaswamy19@gmail.com

C. Rajabhushanam, Professor, Dept. of C.S.E, Bharath Institute of Higher Education and Research, Chennai, Tamilnadu, India. Email: rajabhushanamc.cse@bharathuniv.ac.in system assets to explicit courses using confirmation control.

In order to avoid congestive collapse the congestion control techniques adjust traffic section into a telecommunication system. By lessening the rate of packets, this is regularly cultivated. In this technique the congestion control keeps senders from overpowering the system and to keeps the sender from overpowering the recipient the flow control technique is utilize.

\section{CONGESTION CONTROL MECHANISMS FOR WIRELESS NETWORKS}

There are two protocols in wireless network named TCP and UDP. TCP is the transmission Control Protocol and it is a connection-oriented transport protocol. TCP is used by huge internet applications because TCP gives a reliable byte-stream data transfer. The TCP uses many techniques for congestion control. And every technique manage the dispatching rate by influencing a congestion window (cwnd). This congestion window restrains the quantity of exceptional unacknowledged bytes that are permitted at each time. On the basis of congestion level the size of this window can change. It can be expand or cut. [2].

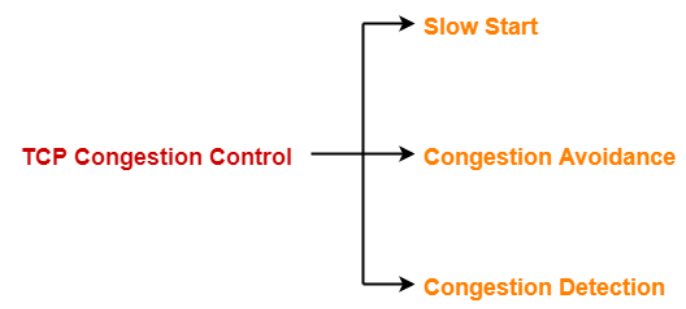

Figure1: phases of TCP's general policy for handling congestion

Congestion control is a significant component of congestion control. The main reason behind the congestion control is to guarantee organize dependability and accomplish a sensibly reasonable appropriation of the system assets among the clients. TCP provide reliable transmission of packets and it also gives the congestion control so we can say that it is a well established protocol. There are many way to proposed changes to the convention to figure out how to tune remote systems for ideal TCP execution. There are several methods to improve the performance of TCP protocol in congestion control. In one method the researchers study the buffer management for TCP with High Speed 
Downlink Packet Access (HSDPA). And after that they assessed the impact of decreasing the lower bound of the retransmit break interim in an environment with fluctuating limit.

When some applications are very strict about timing, it is necessary to use a reliable transport protocol. As of not long ago UDP has been the principle option in contrast to TCP. UDP does not give any reliable transmission, nor congestion control, yet does then again not present any delay in itself. There are anyway worries that expanded utilization of UDP would cause organize unsteadiness and starve the TCP streams that diminish their send rate when rivalry increases. That is the reason another protocol named Datagram Congestion Control Protocol (DCCP) has been introduced. For congestion control presently DCCP uses TFRC and TCP-like protocols. We work on TCP friendly rate control in order to drive the advancement forward.

\section{LITERATURE REVIEW}

In wireless networks congestion control is very serious issue. There are many reason of congestion control include limitation of bandwidth, many to one communication and unpredictable traffic load. As the consequences of congestion control it reduce the performance of the network. There are many possible way to increase the performance of network by improving the techniques of congestion control. Many researchers introduced different methods and approaches for congestion control [4].

In the paper [5] 2013 Dhaneshwar Kumar, Nikita Gupta, Sachin Kumar Saxena, Astha Sharma states that internet providers require QoS for time strict applications and information transmission yet at the season of system congestion, debases QoS. To gives better quality support of a wide range of various traffic system TCP-Friendly congestion control algorithm can used.

In paper [6] 2011 Nguyen Duy Vie, Le Hung Lan, Mai Vinh Du and Tran Xuan Truong describe that in time strict applications congestion lessens the forecasting quality. To control the congestion RED is needed.

In paper [7] 2011 Vijay Raghunathan and A. B. M. Alim AI Islam introduced that for solving the problem of reliable data delivery in wireless mesh network Novel neural network can be utilized. And to create variant like Intelligent TCP or iTCP it incorporate into TCP.

In paper [8] 2007 Wang Xiaoyan and Gong Changqing Zhao Linna describe that there are two reason for data loss during the data transmission are link bit error and network congestion

In paper [9] 2009 Y. Yang, Yanxiang and N. Xiong distributed their work. In order to design an efficient congestion controller the over Congestion Control plan was introduced. And this is based on back-propagation neural network technique. This technique taking care of the issue of dissimilarity between the system assets and the measure of approaching traffic that emerges because of huge engendering delay in information transmission.

In paper [10] 2014 B. Subramani and Dr.T.Karthikeyan distributed their work. In communication network robustness and fairness are significant issues. It needed effective queue management and congestion free techniques.

A few research works were completed by various analysts in the zone of effective correspondence in WSN. A disseminated occasion activated correspondence was done in [11] for time synchronization.

\section{CONGESTION CONTROL TECHNIQUES}

The method which is used to prevent or control the congestion is known as congestion. Congestion control can generally divided in two sub divisions:

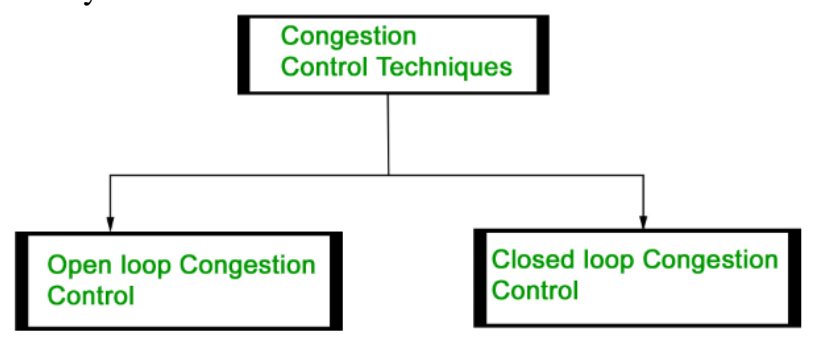

Figure 2: Congestion control techniques

\section{Open Loop Congestion Control}

In this technique the method prevent the congestion before happening. It can be prevent either on source or destination. There are many open loop congestion techniques as follow:

\section{Retransmission Policy:}

In this method packets should be retransmit if some of the packets are corrupted or it is not delivered to the recipient. This type of technique enhances the network congestion. To improve the performance it is require designing the retransmission timer in such a way that it can reduce the congestion.

\section{Window Policy:}

At the sender end the congestion is depend on window type. In the Go-back-n window many packets are resent, in those packets some of the packets successfully get by the receiver. The duplicate packet increases the network congestion. That's why it is require to send only selective packet that have been lost during transmission and Selective repeat window should be used. .

\section{Discarding Policy:}

The router uses a good discarding scheme in which the router discard the less necessary and corrupted packet from the transmission. In this way it reduces the congestion.

\section{Acknowledgment Policy:}

At the receiver end the acknowledgement of receiving a packet or not is also very important and it affect the congestion. There are many techniques are available to prevent the congestion that is related to acknowledgement of packets. In one approach the receiver send the acknowledgement of many packets instead of a single 
packet.

\section{Admission Policy:}

There are using a mechanism to prevent the congestion in admission policy. Before transmitting the packet the switches should check the requirement of a resource of a network flow. If there is any congestion in network then to prevent the congestion router deny establishing a network connection.

\section{Closed Loop Congestion Control}

Closed loop congestion control technique is used to treat or alleviate congestion after it happens. Several techniques are used by different protocols; some of them are:

\section{Backpressure:}

This is the method in which a congested node does not receive packets from upstream node. The upstream node become congested and then it is not receive the packets from higher nodes. This type of method is only use to apply on virtual circuit. And in these circuits each node takes the information of its higher upstream node. We can say that backpressure technique propagate in the reverse order of data flow. It is a node to node congestion control approach.

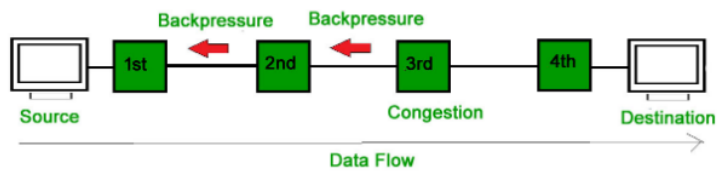

Figure 3: Backpress techniques

The above diagram shows that 3 rd node is congested and it cannot receive the packets that is the reason the 2 nd node is also get congested because output data flows very slowly. Furthermore 1st node is also congested and whole system get slow down..

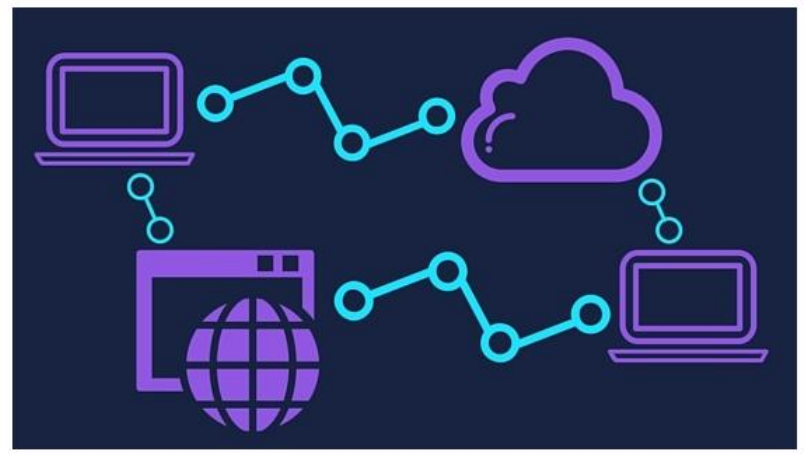

Figure 4: Backpress Routing

\section{Choke Packet Technique:}

In Choke packet method datagram and virtual networks both are used. To inform the congestion a packet is send by a particular node is known as choke packet. Each router assigns the uses to the network and it monitors its resources. When a resource uses more network than its assigned value then the router send a choke packet. This packet is sent by the source and it is the indication of reducing the traffic.

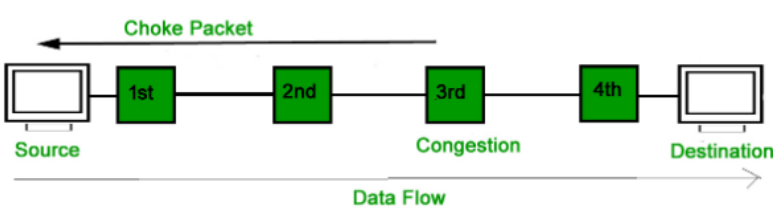

Figure 5: Chock packet techniques

\section{Implicit Signaling:}

In this type of technique the congested node and source does not communicate to each other. If a sender transmit so many data and sender does not get any acknowledge that receiver receive the data then it can assume that there congestion can occurs. So the sources guess about the network congestion.

\section{Explicit Signaling:}

If a node feels that there is congestion in network then the node can send a explicit packet to inform that there is a congestion, this type of signaling known as explicit signaling. As differ from chock packet in explicit signaling with the packet it include signals but the chock packet method include different packets.

There are two type of explicit signaling include forward signaling and backward signaling.

The explicit signaling in which signals are send in the same direction as in congestion is known as forward signaling. In This type of signaling destination have knowledge about the congestion and it apply techniques to avoid the congestion.

The explicit signaling in which signals are send in the reverse direction of congestion is known as backward signaling. Same as the forward signaling destination have knowledge about the congestion and it applies techniques to prevent the congestion.

\section{WAYS TO REDUCE NETWORK CONGESTION}

There is several ways to reduce the network congestion. Some of them are described below:

\section{Network Traffic Monitoring}

If we continuously monitor the traffic of network then we can predict that where the congestion can happen. I $\mathrm{n}$ this way we can find the area of problem and reduce the congestion. 


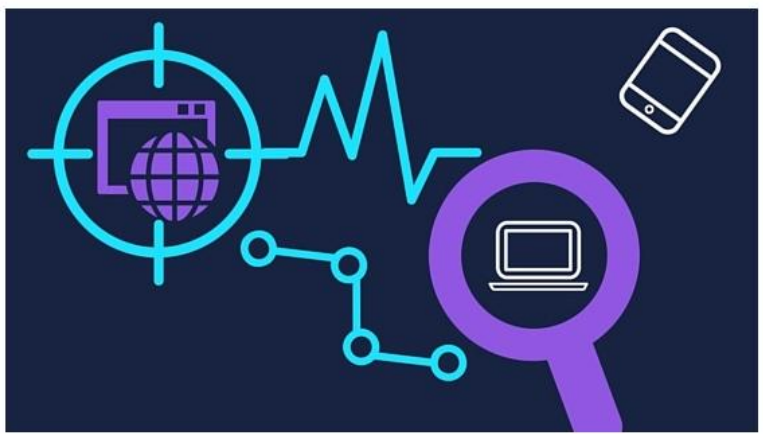

Figure 6: Network treffic monitoring

We have to manage the distribution of network traffic in such a way that we can reduce the network congestion. If it find any congestion then provide some reasonable method to prevent that.

\section{Segmentation of Network}

In network segmentation it divides the whole network in small sub networks. By dividing the network it can manage the network traffic in particular areas and thus it reduce the congestion.

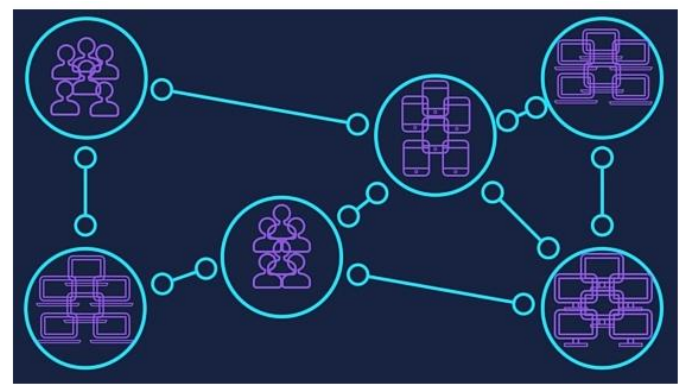

Figure 7: Nework segmenation

\section{Content Delivery Network Use}

By using the content delivery network (CDN) it can provide the global distribution of static content. In CDN contents will place on edge serves. Thus the coming request will reduce and it reduce the possibilities of congestion.

\section{Reconfigure the TCP/IP Settings}

By reconfigure the TCP/IP setting it can reduce the demand of packets. In a network if there is no congestion then packet will deliver fast. If a sender transfer the data fast than receiving the data then there is congestion the receiving end. By reconfigure the TCP/IP setting the transfer of data can be adjust. At the receiving end the computer mage the data properly thus it reduce the congestion.

\section{Prioritize Network Traffic}

By changing the setting of a router it can prevent the congestion. Assigning the priority of traffic is necessary over the network. To set the priority voice over IP (VoIP) settings is used. For proper utilization of bandwidth prioritization of traffic is very useful.

\section{Network Redundancy use}

By using the network redundancy it ensure that if one rote is busy or congested at one time then it can use other route at the same time. So in this way it prevent from packet loss or time delay.

\section{The Datapath.io Solution}

By giving the access to lowest latency network the datapath.io can prevent or reduce the congestion. In provide the path that is depend on global monitoring of the Internet.

\section{ReSUlt}

In this section, we presented our experimental results of TRFC and TCP time out, later sent packets, avoiding congestion control using TCP and TFRC are shown in Figure 8 to Figure 10 respectively

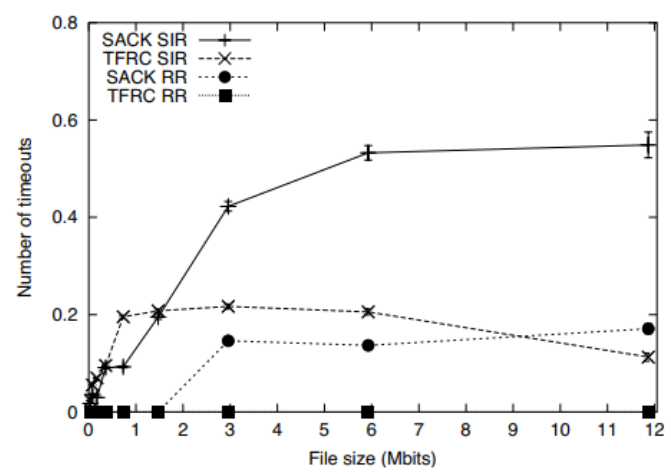

Figure 8: TFRC and TCP timeout [3]

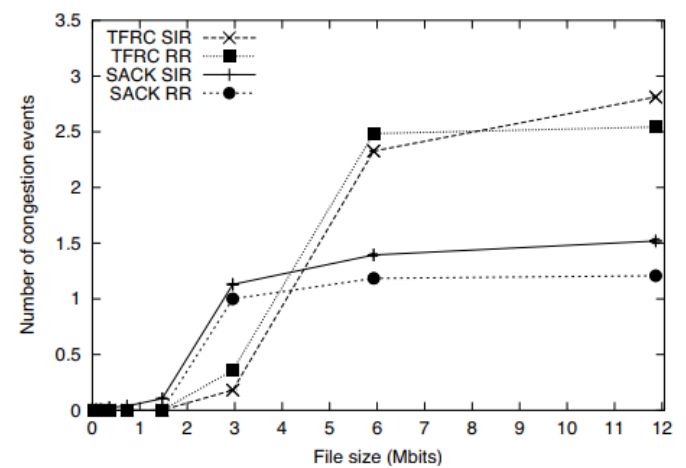

Figure 9: Later sent packet shown that arriving at TFRC receiver [3]

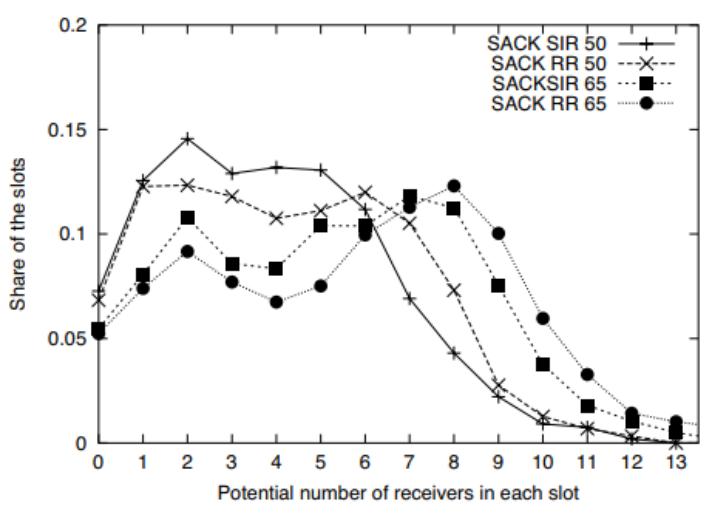


Figure 10: To avoid congestion for TCP no. of potential receivers [3]

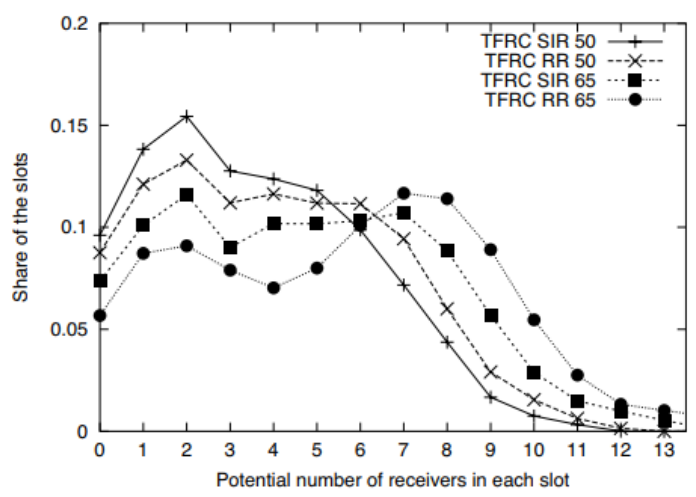

Figure 11: To avoid congestion for TFRC no. of potential receivers [3]

\section{ConClusion}

There are many techniques for congestion control. Our proposed system work efficiently and smoothly design a methodology. In this research work we apply the TCP friendly rate control to control the congestion in wireless network. As shown in result the work compare the TCP and TFRC on different steps.

\section{REFERENCES}

1. (Al-Bahadili, 2012, p. 282) Al-Bahadili, H. (2012). Simulation in computer network design and modeling: Use and analysis. Hershey, PA: IGI Global.

2. K. A. Yadav and S. Kumar, "A review of congestion control mechanisms for wireless networks," 2017 2nd International Conference on Communication and Electronics Systems (ICCES), Coimbatore, 2017, pp. 109-115. doi: 10.1109/CESYS.2017.8321245.

3. Sara Landström, "Congestion Control in Wireless Cellular Networks",

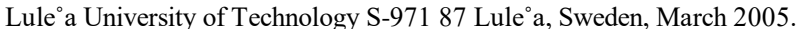

4. Digvijaysinh Basiya, Prof. Deep Patel, "Congestion Control By Using Adaptive Data Rate Technique with High Bandwidth in Wireless Sensor Networks", International Journal on Future Revolution in Computer Science \& Communication Engineering, Volume: 4 Issue: 4.

5. Sachin Kumar Saxena, Dhaneshwar Kumar, Astha Sharma, Nikita Gupta, Radhika Kochhar, "Congestion Control in Wired Network for Heterogonous Resource Using Neural Network", Uttarakhand India, Vol 3, 2013.

6. Tran Xuan Truong, Le Hung Lan, Nguyen Duy Vie and Mai Vinh Du, "Congestion Control in TCP/IP Differentiated Service Network Using Neural Network", IEEE Seventh International Conference on Natural Computation 978-1-4244-9953-3, 2011.

7. A. B. M. Alim AI Islam and Vijay Raghunathan, "Endto-End Congestion Control in Wireless Mesh Networks using a Neural Network", IEEE WCNC, West Lafayette, 2011.

8. Gong Changqing Zhao Linna and Wang Xiaoyan, "Using Neural Network Classifier of Packet Loss Causes to Improve TCP Congestion Control over Ad Hoc Networks", IEEE 2007 International Symposium on Microwave, Antenna, Propagation, and EMC Technologies for Wireless Communications, Tongliao, China, 2007.

9. N. Xiong, Member, Y. Yang, Jing He and Yanxiang, "On Designing QoS for Congestion Control Service Using Neural Network Predictive Techniques", IEEE 1-4244-0134-8, 2006.

10. Dr. T.Karthikeyan and B. Subramani, "A New Congestion Control Algorithm Based on Novel AQM", T.Karthikeyan et al, / (IJCSIT) International Journal of Computer Science and Information Technologies, Vol. 5 (3) , 4440-4443, 2014

11. Zhenping Chen, Dequan Li, Yourui Huang and Chaoli Tang, "Event-triggered communication for time synchronization in WSNs", Neurocomputing, Elsevier, Volume 177, 2016, Pages 416-426. 